\title{
Monitoring breastfeeding rates in Italy
}

\author{
G Banderali, E Riva, S Scaglioni, C Agostoni and M Giovannini \\ Department of Paediatrics, San Paolo Hospital, Milan, Italy
}

Banderali G, Riva E, Scaglioni S, Agostoni C, Giovannini M. Monitoring breastfeeding rates in Italy. Acta Paediatr 2003; Suppl. 441: 6-8. Stockholm. ISSN 0803-5326.

\begin{abstract}
Aim: to determine the rates of initiation and duration of breastfeeding in Italy in 1995 and 1999 , and to examine the adherence to the ten steps to successful breastfeeding recommended by WHO. Methods: Two cohorts of mothers who delivered healthy infants in November $1995(\mathrm{n}=2400)$ or November $1999(\mathrm{n}=3500)$ were interviewed by telephone within 4 wk of delivery when their infants were 3 months of age. Type of breastfeeding was classified according to the WHO definitions. Adherence to the WHO ten steps was evaluated. Results: Initiation and duration of breastfeeding increased during the 1995-1999 period $(p<0.0001)$. The rate of breastfeeding at discharge and when the infants where 3 months of age was $83 \%$ and $42 \%$ in 1995 , and $89 \%$ and $66 \%$ in 1999. The rate of exclusive/predominant breastfeeding at discharge was higher in 1999 than 1995 (78\% vs $72 \%)$.
\end{abstract}

Conclusion: An increase in initiation and duration of breastfeeding through the first 3 months of age occurred in Italy during the 1995-1999 period, but both breastfeeding duration and observance of the WHO's ten steps are not completely satisfactory yet.

Key words: Breastfeeding, WHO's ten steps, Italy

M Giovannini, Department of Paediatrics, San Paolo Hospital, 8 Via A di Rudinì, IT-20142 Milan, Italy (Tel. +3902 81844508, fax. 3902 89150125, e-mail.marcello.giovannini@unimi.it)

Breast milk is the ideal species-specific food for infants. It is easily absorbed, has a low solute load and an increasing availability of minerals, vitamins and proteins. The results of studies indicate that breastfeeding correlates with lower infant mortality and morbidity and confers improved developmental outcome. Promoting breastfeeding is therefore the first concern of paediatricians. The World Health Organization (WHO) has established the ideal breastfeeding duration $(>6 \mathrm{mo}$ ), raising new interest in the conditions that possibly influence breastfeeding practice. Although there has been a reverse trend in the interest in nursing in the West in recent years, studies show that the 6-mo exclusive breastfeeding criterion set by WHO remains to be implemented. In effect, rates of breastfeeding initiation and duration are still lower in many European countries than in the Scandinavian countries, where policies aimed at promoting breastfeeding were implemented many years ago $(1,2)$. Observance of the WHO's ten steps to successful breastfeeding might increase these rates (Table 1). The current prevalence of breastfeeding in Italy as a whole was unknown until 1996: only local or regional surveys existed. The "PUER project 1" was designed to obtain a crosssectional assessment of breastfeeding initiation and duration in Italy and to determine the associated social and biological factors (3).

As part of the "PUER Project 2", which monitors the rates of breastfeeding initiation and duration in Italy, we have evaluated whether any change in the breastfeeding

Table 1. WHO's ten steps to successful breastfeeding (Vallenas C, Savage-King F, Evidence for ten steps to successful breastfeeding. Geneva: WHO Child Health and Development Unit; 1997).

Step 1. Have a written breastfeeding policy that is routinely communicated to all healthcare staff

Step 2. Train all healthcare staff in the skills necessary to implement this policy

Step 3. Inform all women (face-to-face and leaflets) about the benefits and management of breastfeeding

Step 4. Help mothers initiate breastfeeding within $30 \mathrm{~min}$ of delivery

Step 5. Show mothers how to breastfeed and how to maintain lactation (by expressing milk) even if they are likely to be separated from their infants

Step 6. Give newborn infants no food or drink unless "medically" indicated. No promotion of formula milks

Step 7. Practise rooming-in. All mothers should have their infants' cots next to them $24 \mathrm{~h}$ a day

Step 8. $\quad$ Encourage breastfeeding on demand

Step 9. No artificial teats or pacifiers to be given to breastfeeding infants

Step 10. Foster the establishment of breastfeeding support groups and refer mothers to them 
Table 2. Percentage (95\% confidence interval) of breastfeeding initiation in Italy.

\begin{tabular}{lllrr}
\hline Type of breastfeeding & $1995 \%(95 \% \mathrm{CI})$ & $1999 \%(95 \% \mathrm{CI})$ & Change $1999-1995$ & $p$ \\
\hline Any breastfeeding & $83.4(81.6,85.2)$ & $89.0(87.8,90.2)$ & +5.6 & $<0.0001^{*}$ \\
Exclusive or predominant & $71.8(69.6,74.0)$ & $78.0(76.4,79.6)$ & +6.2 & $<0.0001^{*}$ \\
Complementary & $13.5(11.7,15.1)$ & $11.0(9.8,12.2)$ & -2.5 & $0.024^{*}$ \\
\hline
\end{tabular}

* Statistical significance.

Table 3. Percentage (95\% confidence interval) of breastfeeding at three months in Italy

\begin{tabular}{lccrr}
\hline Type of breastfeeding & $1995 \%(95 \% \mathrm{CI})$ & $1999 \%(95 \% \mathrm{CI})$ & Change $1999-1995$ & $p$ \\
\hline Any breastfeeding & $41.8(39.4,44.2)$ & $65.7(63.8,67.6)$ & +22.9 & $<0.0001^{*}$ \\
Exclusive or predominant & $37.3(34.9,39.7)$ & $48.4(46.4,50.4)$ & +11.1 & $<0.0001^{*}$ \\
Complementary & $4.5(3.5,5.5)$ & $17.3(15.8,18.8)$ & +12.8 & $0.0001^{*}$ \\
\hline
\end{tabular}

* Statistical significance.

rate has occurred in Italy in the last 5-y. The aim of this ongoing longitudinal study is also to assess the implementation in Italy of WHO's "ten steps" to successful breastfeeding, evaluating if any of these steps exist mostly in association with the duration of breastfeeding in Italy.

Two cohorts of 2400 and 3500 mother-infant pairs were recruited randomly from all the healthy singleton term newborns during November 1995 and November 1999, respectively. A questionnaire was administered by telephone $1 \mathrm{mo}$ and $3 \mathrm{mo}$ after delivery. Experienced personnel, previously standardized during a training course, carried out the interviews. The participation rates were $66.7 \%$ in 1995 and $70.0 \%$ in 1999. Type of

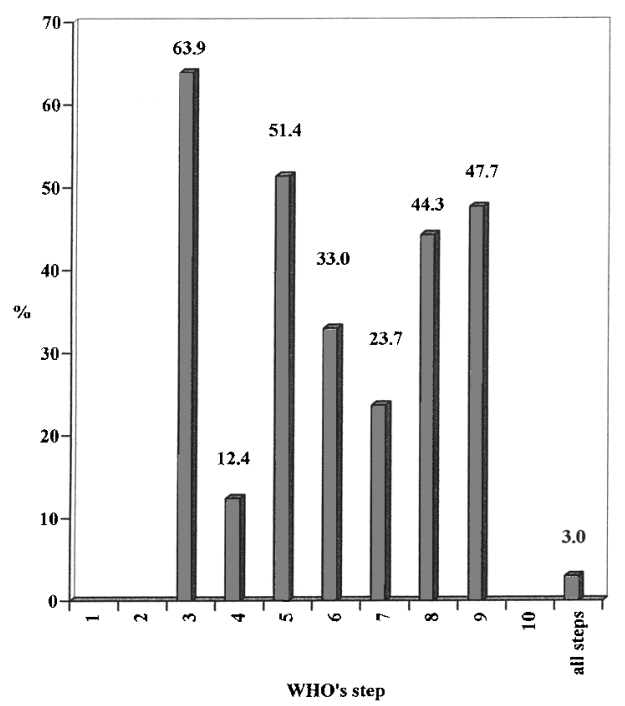

Fig. 1. Percentage of implementation in Italy of the WHO's ten steps to successful breastfeeding. breastfeeding was classified according to the WHO definition. Cox's regression analysis was used to identify the WHO's steps associated with the duration of breastfeeding.

The rate of initiation of breastfeeding was higher in $1999(89.0 \%, 95 \% \mathrm{CI}, 87.8 \%$ to $90.2 \%)$ than in 1995 $(83.4 \%, 95 \%$ CI, $81.6 \%$ to $85.2 \%)(p<0.0001)$. The rate of full (exclusive or predominant) breastfeeding was also higher in 1999 (78.0\%, 95\% CI, 76.4\% to $79.6 \%)$ than in $1995(71.8 \%, 95 \%$ CI, $69.6 \%$ to $74.0 \%)$ $(p<0.0001)$ (Table 2). Three months after delivery, the rate of breastfeeding was $41.8 \%(95 \% \mathrm{CI}, 39.4 \%$ to $42.2 \%$ ) in the 1995 cohort and $65.7 \%$ (95\% CI, $63.8 \%$ to $67.6 \%)$ in the 1999 cohort $(p<0.0001)$. The corresponding rates of full breastfeeding were $37.3 \%$ (95\% CI, $34.9 \%$ to $39.7 \%$ ) and $48.4 \%$ (95\% CI, $46.4 \%$ to $50.4 \%)$, respectively $(p<0.0001)$ (Table 3$)$. Concerning the adherence to WHO's steps, the data from "PUER Project 1" have shown a low percentage, ranging from $12.4 \%$ (95\% CI, $10.8 \%$ to $14.0 \%$ ) of cases for step 4 (breastfeeding within $30 \mathrm{~min}$ of delivery) to $63.9 \%$ (95\% CI, $61.5 \%$ to $66.3 \%$ ) for step 3 (face-to-face information) (Fig. 1).

A multiple logistic analysis disclosed that initiation of breastfeeding was positively associated with mothers being shown how to breastfeed and how to maintain lactation (step 5; odds ratio (OR) 1.5, 95\% CI, 1.1 to 2.2). Using a Cox multiple regression analysis, it was found that the use of artificial teats or pacifiers (step 9) was associated with the stopping of both breastfeeding (rate ratio, RR, 1.2, 95\% CI, 1.0 to 1.3 ) and exclusive/ predominant breastfeeding (RR, 1.3, 95\% CI, 1.2 to 1.5). Promotion of formula milks (step 6) and encouragement to breastfeed on demand (step 8) were not associated with longer duration of exclusive/predominant breastfeeding (RR, 1.1, 95\% CI, 1.0 to 1.3: step 6, RR 1.3, 95\% CI 1.2-1.5: step 8). Initiation of breastfeeding was higher and duration was longer in 
mothers who observed all ten of the WHO steps than in the other mothers (initiation rate $95.8 \%$ vs $84.8 \%$, $p<0.001)$.

In conclusion, the present findings indicate that there has been a significant increase in the rate of breastfeeding in Italy during the past quinquenniuim:

- a $5.6 \%$ increase in the breastfeeding initiation rate

- a $22.9 \%$ increase in the breastfeeding rate at 3 mo of age

Breastfeeding duration and observance of the WHO's ten steps are not completely satisfactory yet. Possible changes related to infant-feeding policies and practice should be identified in order to further support breastfeeding in Italy, according to the WHO recommendations.
Campaigns aimed at educating mothers regarding breastfeeding and discouraging the use of artificial teats and pacifier should be promoted.

\section{References}

1. Zetterström R. Trends in research in infant nutrition, past, present and future. Acta Pædiatr 1994; Suppl 402: 1-3

2. Heiberg Endresen E, Helsing E. Changes in breastfeeding practices in Norwegian maternity wards: national surveys 1973, 1982 and 1991. Acta Pædiatr 1995; 84: 719-24

3. Riva E, Banderali G, Agostoni C, Silano M, Radaelli G, Giovannini M. Factors associated with initiation and duration of breastfeeding in Italy. Acta Pædiatr 1999; 88: 411-5

4. WHO Global Data Bank on Breastfeeding. Breastfeeding: the best start.Geneva: Nutrition Unit, WHO; 1996

5. WHO Nutritional database system. Copenhagen: WHO; 1 May 1998 